\title{
ON THE ZEROS OF CERTAIN COSINE POLYNOMIALS
}

\author{
A. B. MINGARELLI AND S. WANG
}

(Communicated by J. Marshall Ash)

\begin{abstract}
In a recent paper [The first sign change of a cosine polynomial, Proc. Amer. Math. Soc. 111 (1991), 709-716], Zeng studies the location of the first sign change of a cosine polynomial and thereby improves on results by Nulton and Stolarsky [The first sign change of a cosine polynomial, Proc. Amer. Math. Soc. 84 (1982), 55-59]. In this article we present a proof for each one of the four conjectures announced in Zeng's aforementioned paper and also discuss some extensions.
\end{abstract}

\section{INTRODUCTION}

In this note a real cosine polynomial is an expression of the form

$$
f(x)=\sum_{i=1}^{n} a_{i} \cos \lambda_{i} x
$$

where the $a_{i}, i=1, \ldots, n$, are real and $0<\lambda_{1}<\cdots<\lambda_{n}, n \geq 1$.

The study of the location of the first sign change of $f$ was the object of a paper by Zeng [3] in which the following conjectures were presented.

Conjecture 1.1 [3, p. 714]. The first sign change of the cosine polynomial

$$
\cos \lambda_{1} x+\cos \lambda_{2}+\cdots+\cos \lambda_{n} x
$$

belongs to $\left(0, \pi / \lambda_{1}\right)$.

Conjecture 1.2. The first sign change of (1.2) is a decreasing function of $\lambda_{1}$.

Note. Zeng [3] proved the validity of these conjectures for $n \leq 4$ and pointed out that Conjecture 1.1 implies Conjecture 1.2.

Conjecture 1.3. Let $a_{1}=1$ and let $a_{2}, \ldots, a_{n}$ all have the same sign. Then the first sign change of $(1.1)$ belongs to $\left(0, \pi / \lambda_{1}\right)$.

Conjecture 1.4. Under the assumption of Conjecture 1.3, if $f(0) \geq 0$ (resp. $f(0)<0$ ), the first sign change of $f(x)$ is a decreasing (resp. increasing) function of $\lambda_{1}$.

Received by the editors July 29, 1991 and, in revised form, November 27, 1991.

1991 Mathematics Subject Classification. Primary 33B10, 42A05.

Key words and phrases. Cosine polynomial, zeros, almost periodic functions, first sign change. This research was partially funded by a grant from NSERC Canada. 
Note. It is clear that Conjectures 1.3 and 1.4 contain the first two. Furthermore, the status of these conjectures is that they are valid for $n \leq 3$ and (see [3]).

The purpose of this note is to prove Conjectures 1.3 and 1.4 (and so Conjectures 1.1 and 1.2) in their full generality. It is not difficult to see that the relation between Conjectures 1.3 and 1.4 is similar to the one between the first two conjectures, i.e., Conjecture 1.3 implies Conjecture 1.4. So the key is to prove Conjecture 1.3. We originally used techniques drawn from Sturm theory of second-order linear differential equations to prove Conjecture 1.1 for all sufficiently large $\lambda_{i}$. The techniques used in the sequel were inspired by o.d.e.'s but are actually independent. We conclude by giving some extensions of our techniques to almost periodic functions of zero mean value whose Fourier representaion is a pure cosine series with coefficients of one sign.

\section{Proof of the Conjectures}

Without loss of generality, we can always assume that $a_{1}=1, a_{i} \neq 0$, $i=2, \ldots, n$. The basic idea in the proofs that follow is that (1.1) has a sign change in $\left(0, \pi / \lambda_{1}\right)$ provided

$$
\int_{0}^{\pi / \lambda_{1}} q(x) f(x) d x \leq 0
$$

if $f(0)>0$, or

$$
\int_{0}^{\pi / \lambda_{1}} q(x) f(x) d x \geq 0
$$

if $f(0)<0$, for some continuous function $q, q(x)>0$ in $\left(0, \pi / \lambda_{1}\right)$. We divide the proof of the conjectures with a sequence of lemmata.

Lemma 2.1. Let $a_{i}>0, i=2,3, \ldots, n$. Then the first sign change of

$$
f(x)=\cos \lambda_{1} x+a_{2} \cos \lambda_{2} x+\cdots+a_{n} \cos \lambda_{n} x
$$

occurs in $\left(0, \pi / \lambda_{1}\right)$.

Proof. By assumption, $f(0)>0$. Let $q(x)=\sin \lambda_{1} x$. Then

$$
\int_{0}^{\pi / \lambda_{1}} q(x) f(x) d x=\sum_{i=2}^{n} a_{i} \frac{\lambda_{1}}{\lambda_{1}^{2}-\lambda_{i}^{2}}\left(1+\cos \left(\frac{\lambda_{i} \pi}{\lambda_{1}}\right)\right),
$$

as a straightforward calculation will show. It now follows that there holds (2.1), and this completes the proof.

Lemma 2.2. Let $a_{i}<0, i=2,3, \ldots, n$, and $f(0)>0$ where $f$ is defined as in (2.3). Then the first sign change of $f$ occurs in $\left(0, \pi / \lambda_{1}\right)$.

Proof. Since $f(0)>0$, it is clear that $\sum_{i=2}^{n}\left|a_{i}\right|<1$. Furthermore, $f\left(\pi / \lambda_{1}\right) \leq$ $-1+\left|a_{2}\right|+\cdots+\left|a_{n}\right|<0$. The conclusion now follows.

Lemma 2.3. Let $a_{i}<0, i=2,3, \ldots, n$, and $f(0)<0$. Then the first sign change of $f$ occurs in $\left(0, \pi / \lambda_{1}\right)$.

Proof. This is similar to the one given in Lemma 2.1. We choose $q(x)=$ $\sin \lambda_{1} x$, derive (2.4), and note that since $f(0)<0, f(x)$ cannot remain so because the right side of $(2.4)$ is now nonnegative. 
Lemma 2.4. Let $a_{i}<0, i=2,3, \ldots, n$, and $f(0)=0$. Let $\lambda_{2}<\lambda_{3}<\cdots<$ $\lambda_{n}$ be positive odd multiples of (a fixed) $\lambda_{1}$. Then the first sign change of $f$ occurs in $\left(0, \pi / \lambda_{1}\right)$.

Proof. Note that $f(0)=0$ implies that $a_{2}+\cdots+a_{n}=-1$. Also $f^{\prime}(0)=0$. Moreover,

$$
\begin{aligned}
f^{\prime \prime}(0) & =-\lambda_{1}^{2}-a_{2} \lambda_{2}^{2}-a_{3} \lambda_{3}^{2}-\cdots-a_{n} \lambda_{n}^{2} \\
& =\left(a_{2}+\cdots+a_{n}\right) \lambda_{1}^{2}-a_{2} \lambda_{2}^{2}-a_{3} \lambda_{3}^{2}-\cdots-a_{n} \lambda_{n}^{2} \\
& =a_{2}\left(\lambda_{1}^{2}-\lambda_{2}^{2}\right)+\cdots+a_{n}\left(\lambda_{1}^{2}-\lambda_{n}^{2}\right) .
\end{aligned}
$$

Thus $f^{\prime \prime}(0)>0$, by our assumptions, and so $f(x)>0$ in a right neighborhood of $x=0$. Next $f\left(\pi / \lambda_{1}\right)=0, f^{\prime}\left(\pi / \lambda_{1}\right)=0$ since $\cos \left(\lambda_{i} \pi / \lambda_{1}\right)=-1$ and $\sin \left(\lambda_{i} \pi / \lambda_{1}\right)=0$. Furthermore,

$$
f^{\prime \prime}\left(\pi / \lambda_{1}\right)=\lambda_{1}^{2}+a_{2} \lambda_{2}^{2}+\cdots+a_{n} \lambda_{n}^{2}
$$

and so by $(2.5), f^{\prime \prime}\left(\pi / \lambda_{1}\right)=-f^{\prime \prime}(0)<0$. Hence we see that $f(x)$ is negative in a left neighborhood of $x=\pi / \lambda_{1}$. Thus $f(x)$ changes sign in $\left(0, \pi / \lambda_{1}\right)$.

Note. It is easy to check that $f\left(\pi /\left(2 \lambda_{1}\right)\right)=0$, i.e., the point $x=\pi /\left(2 \lambda_{1}\right)$ is a zero of $f$ in Lemma 2.4. It also appears as if a sign change always occurs there. The idea is that it is clear that $f^{(2 k)}\left(\pi /\left(2 \lambda_{1}\right)\right)=0$ for $k=$ $0,1, \ldots$. On the other hand, if $f^{\prime}\left(\pi /\left(2 \lambda_{1}\right)\right) \neq 0$, then a sign change must occur there. If $f^{\prime}\left(\pi /\left(2 \lambda_{1}\right)\right)=0$, we simply perform more differentiations. Since $f$ is a (complex) analytic function, there must exist an integer $k$ for which $f^{(2 k+1)}\left(\pi /\left(2 \lambda_{1}\right)\right) \neq 0$, and consequently $f$ changes its sign there.

Lemma 2.5. Let $a_{i}<0, i=2,3, \ldots, n$, and $f(0)=0$. Then the first sign change of $f$ occurs in $\left(0, \pi / \lambda_{1}\right)$.

Proof. An earlier calculation (Lemma 2.4) shows that $f^{\prime}(0)=0, f^{\prime \prime}(0)>0$, and $f$ is positive in some right neighborhood of $x=0$. Now $f(0)=0$ if and only if $1+a_{2}+\cdots+a_{n}=0$ or $\left|a_{2}\right|+\cdots+\left|a_{n}\right|=1$. Next

$$
f\left(\pi / \lambda_{1}\right) \leq-1+\left|a_{2}\right|+\cdots+\left|a_{n}\right|=0
$$

and so $f\left(\pi / \lambda_{1}\right) \leq 0$. If $f\left(\pi / \lambda_{1}\right)<0$, we immediately obtain the conclusion. On the other hand, if $f\left(\pi / \lambda_{1}\right)=0$ then, by definition,

$$
a_{2} \cos \left(\lambda_{2} \pi / \lambda_{1}\right)+\cdots+a_{n} \cos \left(\lambda_{n} \pi / \lambda_{1}\right)=1 .
$$

Using the relation $1+a_{2}+\cdots+a_{n}=0$ we can rewrite the last display as

$$
a_{2}\left(1+\cos \left(\lambda_{2} \pi / \lambda_{1}\right)\right)+\cdots+a_{n}\left(1+\cos \left(\lambda_{n} \pi / \lambda_{1}\right)\right)=0 .
$$

Since $a_{i}<0$, by assumption, and the fact that every term is of the same sign, it follows that

$$
1+\cos \left(\lambda_{i} \pi / \lambda_{1}\right)=0
$$

for each $i=2, \ldots, n$. Thus each $\lambda_{i}$ must be a positive odd multiple of $\lambda_{1}$. The conclusion now follows upon an application of Lemma 2.4.

Theorem 2.1 (Conjecture 1.3). Let $a_{2}, \ldots, a_{n}$ all have the same sign and let $0<\lambda_{1}<\cdots<\lambda_{n}$. Then the first sign change of $f$, defined as in (2.3), occurs in $\left(0, \pi / \lambda_{1}\right)$.

Proof. This is essentially contained in the last five lemmata. Basically, the result is proved if we can prove it for $f$ such that $a_{i}>0$ for $i=2, \ldots, n$ (Lemma 
2.1) and for $f$ such that $a_{i}<0$ for $i=2, \ldots, n$. In the latter case, the result follows provided we can show it for those $f$ with $f(0)>0$ (Lemma 2.2), $f(0)=0$ (Lemma 2.5), and $f(0)<0$ (Lemma 2.3). The proof is complete.

Theorem 2.2 (Conjecture 1.4). Under the assumptions of Theorem 2.1 , if $f(0) \geq$ 0 (resp. $f(0)<0$ ) then the first sign change of $f$ is a decreasing (resp. increasing) function of $\lambda_{1}$.

Proof. It is similar to Zeng's proof [3] and we omit it.

\section{EXTENSIONS}

We refer the reader to [1] for notions regarding real (Bohr) almost periodic functions. Let $\left(a_{i}\right)$ be a real sequence and $0<\lambda_{1}<\cdots<\lambda_{n}<\cdots, \lambda_{j} \rightarrow \infty$ as $j \rightarrow \infty$. We assume that the Fourier series

$$
\sum_{n=1}^{\infty} a_{n} \cos \lambda_{n} x
$$

converges uniformly on $R$-then it represents an even almost periodic function of mean value equal to zero (or mean zero). Conversely, every even almost periodic function of mean zero is the uniform limit of partial sums of a series of the form (3.1) (Bohr approximation theorem).

Now every (nontrivial) real almost periodic function of mean zero must change sign on $R$ [1]. Hence there must be a smallest positive (and a largest negative) sign change for said function.

Consider the function

$$
f(x)=\cos \lambda_{1} x+\sum_{n=2}^{\infty} a_{n} \cos \lambda_{n} x
$$

where the $a_{n}$ 's either form a positive or a negative sequence and the $\lambda_{n}$ 's are as above. Under appropriate conditions on the series we see that all the lemmata of $\S 2$ are valid in this setting and so one can prove

Theorem 3.1. Let $f$ be an even almost periodic function of mean zero whose Fourier representation, as given by (3.2) converges absolutely (and uniformly) on $R$. If the sequence of $a_{i}$ 's is of one sign, then the first positive sign change of $f$ occurs in $\left(0, \pi / \lambda_{1}\right)$.

Remark. An analog of Theorem 2.2 also exists in this setting and can be readily formulated.

\section{REFERENCES}

1. A. Besicovitch, Almost periodic functions, Dover, New York, 1954.

2. J. D. Nulton and K. B. Stolarsky, The first sign change of a cosine polynomial, Proc. Amer. Math. Soc. 84 (1982), 55-59.

3. J. Zeng, The first sign change of a cosine polynomial, Proc. Amer. Math. Soc. 111 (1991), 709-716.

Department of Mathematics and Statistics, Carleton University, Ottawa, Ontario, CANADA KIS 5B6

E-mail address: ANGELO_MINGARELLI@CARLETON.CA 